\title{
Dinâmica da comunidade lenhosa de um Cerrado Típico na região Nordeste do Estado de Mato Grosso, Brasil
}

\author{
Henrique Augusto Mews ${ }^{1,2,4}$, Beatriz Schwantes Marimon ${ }^{1}$, Leandro Maracahipes ${ }^{1}$, \\ Daniel David Franczak ${ }^{3}$ \& Ben Hur Marimon-Junior ${ }^{1}$ \\ ${ }^{1}$ Pós-graduação em Ecologia e Conservação, Universidade do Estado de Mato Grosso - UNEMAT, \\ CP 08, CEP 78690-000, Nova Xavantina, MT, Brasil \\ ${ }^{2}$ Pós-graduação em Ciências Florestais, Universidade de Brasília-UnB, \\ CP 4357, CEP 70919-970, Brasília, DF, Brasil \\ ${ }^{3}$ Pós-graduação em Botânica, Universidade de Brasília - UnB, \\ CP 4457, CEP 70919-970, Brasília, DF, Brasil \\ ${ }^{4}$ Autor para correspondência: Henrique Augusto Mews, e-mail: henriquemews@gmail.com
}

MEWS, H.A., MARIMON, B.S., MARACAHIPES, L., FRANCZAK, D.D. \& MARIMON-JUNIOR, B.H. Dynamics of the woody community of a typical cerrado in Northeastearn Mato Grosso, Brazil. Biota Neotrop. 11(1): http://www.biotaneotropica.org.br/v11n1/en/abstract?article+bn01511012011.

\begin{abstract}
The objective of the study was to investigate changes in the woody plant community in a four-year period. It was established 50 permanent plots of $10 \times 10 \mathrm{~m}$ and all individuals with diameter above ground $30 \mathrm{~cm}$ $\left(\right.$ DAG $\left._{30 \mathrm{~cm}}\right) \geq 5 \mathrm{~cm}$ were sampled in 2002 and 2006. In the inventory of January 2002, were found 945 individuals belonging to 77 species, 65 genera and 35 families and in January 2006, were 1,106 individuals, 80 species, 66 genera and 36 families. Community species diversity and diameter structure did not change within the period. Mortality rates $\left(4.01 \%\right.$ year $\left.^{-1}\right)$ and basal area losses $\left(0.68 \%\right.$ year $\left.^{-1}\right)$ were offset by recruitment $\left(6.67 \%\right.$ year $\left.^{-1}\right)$ and gain $\left(2.26 \%\right.$ year $\left.^{-1}\right)$, indicating community maintenance as stable apparently. The periodic annual increment (PAI) of the community was $0.31 \mathrm{~cm}^{\text {year }}{ }^{-1}$, being overcome by the PAI of Euplassa inaequalis, Kielmeyera rubriflora and Byrsonima coccolobifolia ( $0.72,0.49$ and $0.47 \mathrm{~cm}_{\text {year }}{ }^{-1}$, respectively), indicating the growth potential of such species as important for recovery strategies on degraded areas. The absence of fire during the study period may have been the main factor behind the dynamics of the vegetation of the studied cerrado, favoring the establishment of some woody species and providing increase in density and biomass.
\end{abstract}

Keywords: savanna, structural changes, temporary alterations, mortality, recruitment.

MEWS, H.A., MARIMON, B.S., MARACAHIPES, L., FRANCZAK, D.D. \& MARIMON-JUNIOR, B.H. Dinâmica da comunidade lenhosa de um Cerrado Típico na região Nordeste do Estado de Mato Grosso, Brasil. Biota Neotrop. 11(1): http://www.biotaneotropica.org.br/v11n1/pt/abstract?article+bn01511012011.

Resumo: O objetivo do trabalho foi analisar as mudanças na comunidade lenhosa em um período de quatro anos. Foram estabelecidas 50 parcelas permanentes de $10 \times 10 \mathrm{~m}$ onde foram amostrados todos os indivíduos com diâmetro a $30 \mathrm{~cm}$ acima do solo (DAS $30 \mathrm{~cm}$ ) $\geq 5 \mathrm{~cm}$ em 2002 e em 2006. No inventário de janeiro de 2002, foram encontrados 945 indivíduos pertencentes a 77 espécies, 65 gêneros e 35 famílias ao passo que em janeiro de 2006 foram 1.106 indivíduos distribuídos em 80 espécies, 66 gêneros e 36 famílias. A diversidade de espécies e a estrutura diamétrica da comunidade não diferiram no período. As taxas de mortalidade $\left(4,01 \%\right.$ ano $\left.{ }^{-1}\right)$ e de perda de área basal $\left(0,68 \%\right.$ ano $\left.^{-1}\right)$ foram compensadas pelas taxas de recrutamento $\left(6,67 \%\right.$ ano $\left.{ }^{-1}\right)$ e de ganho de área basal $\left(2,26 \%\right.$ ano $\left.^{-1}\right)$, indicando a manutenção da comunidade como aparentemente estável. O incremento periódico anual (IPA) da comunidade foi de $0,31 \mathrm{~cm}^{-1}$, sendo superado pelo IPA de Euplassa inaequalis, Kielmeyera rubriflora e Byrsonima coccolobifolia $\left(0,72,0,49\right.$ e $0,47 \mathrm{~cm}^{-1} \mathrm{ano}^{-1}$, respectivamente), indicando o potencial de crescimento de tais espécies como importante para estratégias de recuperação de áreas degradadas. A ausência de fogo no período estudado pode ter sido o fator responsável pela dinâmica da vegetação do cerrado estudado, favorecendo o estabelecimento de algumas espécies lenhosas e proporcionando aumento em densidade e biomassa.

Palavras-chave: savana, mudanças estruturais, alterações temporais, mortalidade, recrutamento. 


\section{Introdução}

A grande riqueza de espécies, associada aos elevados níveis de endemismo, concedeu ao bioma Cerrado o título de uma das savanas mais ricas e diversas do mundo (Silva \& Bates 2002, Walter et al. 2008). Em contrapartida, as taxas de desmatamento deste bioma têm sido muito elevadas em relação aos esforços para sua conservação, tornando-o altamente ameaçado (Klink \& Machado 2005). Estima-se que atualmente existam aproximadamente $51,5 \%$ de áreas nativas remanescentes do Cerrado e sua perda, principalmente pelo desmatamento para a agropecuária, continua acelerada (MMA 2009). Além disso, as áreas remanescentes estão sob efeito de fatores de degradação ambiental, como invasão biológica por espécies exóticas, mudanças no regime de fogo e utilização de áreas naturais para pastagem (Klink \& Machado 2005, Oliveira 2005, Pivello 2005). Essas alterações, ocorridas principalmente nas últimas quatro décadas, têm levado à degradação dos ecossistemas e à perda de biodiversidade no bioma Cerrado (Ratter et al. 1997, Klink \& Machado 2005).

Silva \& Bates (2002) estimam que cerca de $24 \%$ da área do Cerrado é representada por um mosaico de transição savana-floresta e estas zonas de ecótono podem representar importantes oportunidades para a especiação. A região Nordeste do Estado de Mato Grosso apresenta uma destas zonas de tensão ecológica caracterizada pela transição entre os dois maiores biomas brasileiros, o Cerrado e a Floresta Amazônica (Ratter et al. 2003, Marimon et al. 2006). Ratter et al. (2003) apontaram a ocorrência de elevada diversidade para as fitofisionomias da região e sugerem a formação de um grupo fitogeográfico particular nesta zona de transição.

No bioma Cerrado, a maioria dos estudos que enfocam a vegetação está relacionada aos levantamentos florísticos e fitossociológicos, a padrões de distribuição de espécies e aos aspectos ecológicos do fogo, sendo que pouco ainda se sabe sobre o crescimento e a produção de biomassa da vegetação deste complexo ecossistema (Arce et al. 2000). Assim, ressalta-se a necessidade de pesquisas em dinâmica vegetacional (Arce et al. 2000, Felfili et al. 2000, Aquino et al. 2007a, b), pois tais estudos podem subsidiar iniciativas de conservação (Schiavini et al. 1998, Aquino et al. 2007b), manejo e recuperação de áreas degradadas (Aquino et al. 2007a), além de permitir a avaliação das causas e consequências das mudanças ocorridas em função do tempo na estrutura das populações, permitindo ampliar o conhecimento disponível sobre as espécies e maximizar o sucesso destas quando utilizadas na restauração de áreas degradadas (Schiavini et al. 1998). A partir das informações fornecidas pelos estudos de dinâmica pode-se realizar avaliação detalhada dos padrões espaciais de mortalidade, recrutamento, crescimento e regeneração, que embasarão o entendimento dos processos ecológicos da comunidade (Arce et al. 2000, Corrêa \& Van Den Berg 2002, Henriques \& Hay 2002, Aquino et al. 2007b).

O objetivo do presente estudo foi analisar as mudanças na composição florística, na diversidade e na estrutura da vegetação lenhosa de uma área de Cerrado Típico (sensu Ribeiro \& Walter 2008) localizado na zona de transição entre os biomas Cerrado e Floresta Amazônica em um período de quatro anos (2002 a 2006).

\section{Material e Métodos}

O estudo foi realizado em uma área de Cerrado Típico no

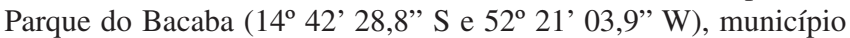
de Nova Xavantina, região Nordeste de Mato Grosso, situada na região de transição entre os biomas Cerrado e Floresta Amazônica (Marimon et al. 2006). O Parque é uma unidade de conservação municipal que ocupa uma área de 492 hectares, dos quais 154 ha são de Cerrado Típico (Abad \& Marimon 2008). A altitude média no Parque é de 348,5 m e o solo, do tipo Latossolo Amarelo, é predominantemente distrófico, ácido $(\mathrm{pH}<5,0)$, álico, profundo, bem drenado, de textura média a arenosa e com reduzidos níveis de cálcio e magnésio (Marimon-Junior \& Haridasan 2005). O Cerrado Típico é um subtipo de Cerrado sentido restrito e apresenta vegetação predominantemente arbóreo-arbustiva, com cobertura arbórea de 20 a $50 \%$ e alturas entre 3 e $6 \mathrm{~m}$ (Ribeiro \& Walter 2008).

O clima da região é do tipo Aw, segundo o sistema de classificação de Köppen (Silva et al. 2008), com um período seco entre maio e setembro e outro chuvoso entre outubro e março. A precipitação média anual, segundo dados coletados na Estação Meteorológica ( $9^{\circ}$ Distrito de Meteorologia, Ministério da Agricultura) localizada no Parque do Bacaba, é de $1.536 \mathrm{~mm}$, calculado para um período de 15 anos (1990 a 2005), com médias de temperaturas anual em torno de $25,5^{\circ} \mathrm{C}$ (Marimon \& Felfili 2006).

No primeiro inventário, realizado em janeiro de 2002, foram estabelecidas 50 parcelas permanentes de $10 \times 10 \mathrm{~m}$ onde todos os indivíduos vivos que apresentaram diâmetro a $30 \mathrm{~cm}$ do solo (DAS $30 \mathrm{~cm}$ ) $\geq 5 \mathrm{~cm}$ foram registrados e medidos em altura e DAS (Marimon-Junior \& Haridasan 2005), recebendo placas de alumínio numeradas. O segundo inventário foi realizado em janeiro de 2006, ocasião na qual todos os indivíduos foram remedidos e os recrutas (indivíduos vivos que atingiram o critério mínimo de inclusão) incluídos. Os resultados do primeiro inventário estão disponíveis em Marimon-Junior \& Haridasan (2005). Em agosto de 2001 foi registrada a ocorrência de incêndio florestal na área, atingindo o Cerrado Típico avaliado no presente estudo (Abad \& Marimon 2008).

Foram coletadas amostras de material botânico das espécies para identificação e inclusão no Herbário NX, Universidade do Estado de Mato Grosso, Campus de Nova Xavantina. As espécies foram identificadas por meio de consultas a especialistas ou por comparações em herbários (NX e UB). O sistema de classificação adotado foi o “Angiosperm Phylogeny Group" (APG 2009) e a revisão e atualização dos nomes dos táxons foram realizadas a partir do banco de dados do "Missouri Botanical Garden" (Mobot 2010).

Foram calculados para a comunidade, com base nas coletas de ambos os inventários, os parâmetros fitossociológicos (Müeller-Dombois \& Ellenberg 1974), o índice de diversidade de espécies de ShannonWiener (H', na base neperiana), a equabilidade de Pielou (J') (Brower \& Zar 1977, Ludwing \& Reynolds 1988) e o incremento periódico anual (IPA) (Encinas et al. 2005). Para avaliar as mudanças estruturais da comunidade foram calculadas as taxas anuais médias de mortalidade $\left(\mathrm{M}=\left\{1-\left[\left(\mathrm{N}_{0}-\mathrm{m}\right) / \mathrm{N}_{0}\right]^{1 / \mathrm{t}}\right\} \times 100\right)$ e recrutamento $\left(\mathrm{R}=\left[1-\left(1-\mathrm{r} / \mathrm{N}_{\mathrm{t}}\right)^{1 / t}\right] \times 100\right)$ em termos de número de indivíduos e área basal, bem como a de perda $\left(\mathrm{P}=\left\{1-\left[\left(\mathrm{AB}_{0}-\mathrm{AB}_{\mathrm{m}}+\mathrm{AB}_{\mathrm{d}}\right) / \mathrm{AB}_{0}\right]^{1 / t}\right\} \times 100\right)$ e ganho $\left(\mathrm{G}=\left\{1-\left[1-\left(\mathrm{AB}_{\mathrm{r}}+\mathrm{AB}_{\mathrm{g}}\right) / \mathrm{AB}_{\mathrm{t}}\right]^{1 / \mathrm{t}}\right\} \times 100\right)$ em área basal, onde $t$ é o tempo transcorrido entre os dois levantamentos, $N_{o}$ e $N_{t}$ são as contagens inicial e final de indivíduos, $m$ e $r$ são o número de indivíduos mortos e recrutas, $A B_{0}$ e $A B_{t}$ são as áreas basais inicial e final, $A B_{m}$ e $A B_{r}$ são as áreas basais dos indivíduos mortos e dos recrutas, $A B_{d}$ é o decremento (quebra ou perda parcial de tronco) e $A B_{g}$ é o incremento em área basal dos sobreviventes, conforme sugerido por Sheil et al. (1995, 2000).

Adicionalmente, foi calculada a reposição em número de indivíduos e em área basal $\left(\mathrm{R}=\left(\mathrm{T}_{1 / 2}+\mathrm{T}_{2}\right) / 2\right)$, as taxas de mudança líquida em número de indivíduos $\left(\mathrm{Ch}_{\mathrm{N}}=\left[\left(\mathrm{N}_{/} / \mathrm{N}_{0}\right)^{1 / \mathrm{t}}-1\right] \times 100\right)$ e área basal $\left(\mathrm{Ch}_{\mathrm{AB}}=\left[\left(\mathrm{AB}_{\mathrm{t}} / \mathrm{AB}_{0}\right)^{1 / \mathrm{t}}-1\right] \times 100\right)($ Korning \& Balslev 1994), o tempo de meia vida, o tempo de duplicação (Swaine \& Lieberman, 1987) e a estabilidade (Korning \& Balslev 1994). As variações temporais observadas nas classes diamétricas foram determinadas contabilizando-se o número de indivíduos que permaneceu, morreu, recrutou, imigrou (ingrowth) e emigrou (outgrowth) nas classes (Lieberman et al. 1985). 
Para a confecção dos histogramas de diâmetro, os intervalos de classes foram calculados de acordo com Bonini \& Bonini (1972) por meio da fórmula $\mathrm{A} / \mathrm{K}$, onde $A$ representa a amplitude dos valores de diâmetro e $K$ representa uma constante definida pelo algoritmo de Sturges, cuja fórmula é $1+3,3 \times \log _{10} \mathrm{n}$, onde $n$ é o número total de indivíduos amostrados. Para todas as classes de diâmetro foram elaboradas distribuições de indivíduos e de área basal e calculados os valores absolutos de mortalidade, recrutamento, ingresso, egresso e mudança temporal. Os valores de H' de ambos os inventários foram comparados por meio do Teste $t$ de Hutcheson (Zar 1999). Para avaliar se houve diferença significativa nas classes de diâmetro entre 2002 e 2006 foi empregado o teste de Kolmogorov-Smirnov (Siegel \& Castellan-Júnior 1988). Para verificar a existência de associação entre a mortalidade (densidade de indivíduos e área basal dos indivíduos mortos), o recrutamento (densidade de indivíduos e área basal dos indivíduos recrutados) e o IPA com as classes de diâmetro foram aplicadas correlações de Spearman (Zar 1999).

\section{Resultados}

\section{Composição florística e diversidade de espécies}

A composição florística apresentou algumas mudanças no período de quatro anos. No primeiro inventário (2002), foram registradas 77 espécies, 65 gêneros e 35 famílias (Marimon-Junior \& Haridasan 2005), ao passo que em 2006 foram registradas 80 espécies, 66 gêneros e 35 famílias (Tabela 1). Entre os anos inventariados, três espécies desapareceram (Antonia ovata Pohl, Myrcia sellowiana O. Berg e Palicourea rigida Kunth) e seis surgiram (Cybianthus detergens Mart., Cordiera sessilis (Vell.) Kuntze, Odontadenia lutea (Vell.) Markgr., Byrsonima verbascifolia (L.) DC., Erythroxylum engleri O. E. Schulz. e E. testaceum Peyr.), resultando em acréscimo líquido de três espécies e um gênero (Tabela 1). As famílias com os maiores números de espécies não mudaram de posição entre os dois levantamentos, merecendo destaque Fabaceae e Vochysiaceae, com 14 e seis espécies, respectivamente. Apesar das mudanças que ocorreram na riqueza e composição florística, os valores de H' de $2002(3,78)$ e de $2006(3,78)$ não diferiram pelo Teste $t$ de Hutcheson $(t=0,09, p>0,05)$ e a equabilidade praticamente não mudou entre os inventários $(2002=0,87$ e $2006=0,86)$.

\section{Estrutura da comunidade}

O número de indivíduos registrados aumentou de 945 em 2002 para 1.106 em 2006, representando um ganho líquido de 161, como resultado da morte de 106 e do recrutamento de 267 indivíduos, o que refletiu em maior área basal total da comunidade em 2006 $\left(14,54 \mathrm{~m}^{2}\right)$ em relação a $2002\left(12,52 \mathrm{~m}^{2}\right)$. Os indivíduos mortos e o decremento (troncos quebrados ou perda parcial dos sobreviventes) da comunidade representaram perdas líquidas de $0,34 \mathrm{~m}^{2}$ e $0,05 \mathrm{~m}^{2}$, respectivamente, refletindo em taxa anual média de perda de área basal de $0,68 \%$ ano $^{-1}$ (Tabela 2). O recrutamento e o incremento em área basal dos sobreviventes contribuíram com $0,79 \mathrm{~m}^{2}$ e $0,84 \mathrm{~m}^{2}$, respectivamente, para o aumento líquido da área basal da comunidade, resultando em uma taxa anual média de ganho de área basal de $2,27 \%$ ano $^{-1}$ (Tabela 2).

Tabela 1. Famílias e espécies (com seus respectivos parâmetros fitossociológicos) registradas em um Cerrado Típico no Parque do Bacaba, Nova Xavantina, MT no ano de 2006. N - número de indivíduos; DR - densidade relativa; FR - frequência relativa; DoR - dominância relativa; VI - valor de importância. Espécies em ordem decrescente de VI. Valores relativos em \%. **Espécies amostradas apenas em 2006.

Table 1. Families and species (with respective phytosociological parameters) sampled in a typical cerrado of the Bacaba Park, Nova Xavantina, Mato Grosso State, in 2006. N - number of individuals; DR - relative density; FR - relative frequency; DoR - relative dominance; VI - importance value. Species in decrescent order of VI. Relative values in \%. ** Species sampled only in 2006.

\begin{tabular}{|c|c|c|c|c|c|c|}
\hline Espécies & Famílias & $\mathbf{N}$ & DR & FR & DoR & VI \\
\hline Qualea parviflora Mart. & Vochysiaceae & 116 & 10,55 & 5,49 & 11,5 & 27,49 \\
\hline Davilla elliptica A. St.-Hil. & Dilleniaceae & 76 & 6,87 & 5,76 & 5,46 & 18,09 \\
\hline Roupala montana Aubl. & Proteaceae & 47 & 4,25 & 3,27 & 6,96 & 14,48 \\
\hline Syagrus flexuosa (Mart.) Becc. & Arecaceae & 65 & 5,88 & 3,8 & 3,8 & 13,47 \\
\hline Aspidosperma tomentosum Mart. & Apocynaceae & 43 & 3,89 & 4,06 & 2,96 & 10,91 \\
\hline Qualea grandiflora Mart. & Vochysiaceae & 37 & 3,35 & 3,27 & 3,98 & 10,59 \\
\hline Byrsonima pachyphylla A. Juss. & Malpighiaceae & 41 & 3,71 & 3,53 & 1,77 & 9,02 \\
\hline Qualea multiflora Mart. & Vochysiaceae & 35 & 3,16 & 3,27 & 1,73 & 8,17 \\
\hline Guapira graciliflora (Schmidt) Lundell & Nyctaginaceae & 41 & 3,71 & 1,31 & 2,53 & 7,55 \\
\hline Euplassa inaequalis (Pohl) Engl. & Proteaceae & 13 & 1,18 & 1,31 & 4,87 & 7,35 \\
\hline Vochysia rufa Mart. & Vochysiaceae & 24 & 2,17 & 2,49 & 2,38 & 7,04 \\
\hline Magonia pubescens A. St.-Hil. & Sapindaceae & 26 & 2,35 & 2,49 & 2,11 & 6,94 \\
\hline Astronium fraxinifolium Schott ex Spreng. & Anacardiaceae & 23 & 2,08 & 2,49 & 2,32 & 6,89 \\
\hline Kielmeyera rubriflora Cambess. & Clusiaceae & 20 & 1,81 & 2,36 & 2,61 & 6,78 \\
\hline Syagrus comosa (Mart.) Mart. & Arecaceae & 29 & 2,62 & 2,36 & 1,74 & 6,71 \\
\hline Myrcia lanuginosa O. Berg. & Myrtaceae & 31 & 2,8 & 2,62 & 1,15 & 6,57 \\
\hline Ouratea hexasperma (A. St.-Hil.) Baill. & Ochnaceae & 23 & 2,08 & 1,96 & 1,81 & 5,85 \\
\hline Pseudobombax longiflorum (Mart. \& Zucc.) A. Robyns & Malvaceae & 18 & 1,63 & 2,09 & 1,46 & 5,18 \\
\hline Kielmeyera speciosa A. St.-Hil. & Clusiaceae & 17 & 1,54 & 2,09 & 1,41 & 5,04 \\
\hline Lafoensia pacari A. St.-Hil. & Lythraceae & 20 & 1,81 & 2,09 & 1,09 & 4,99 \\
\hline Dalbergia miscolobium Benth. & Fabaceae & 18 & 1,63 & 1,96 & 1,38 & 4,97 \\
\hline Byrsonima coccolobifolia Kunth & Malpighiaceae & 18 & 1,63 & 1,83 & 1,44 & 4,9 \\
\hline Strychnos pseudoquina A. St.-Hil. & Loganiaceae & 11 & 0,99 & 1,31 & 2,58 & 4,88 \\
\hline Dimorphandra mollis Benth. & Fabaceae & 14 & 1,27 & 1,83 & 1,45 & 4,55 \\
\hline Bowdichia virgilioides Kunth & Fabaceae & 12 & 1,08 & 1,44 & 2,01 & 4,54 \\
\hline Terminalia argentea Mart. & Combretaceae & 11 & 0,99 & 1,31 & 2,15 & 4,45 \\
\hline
\end{tabular}


Tabela 1. continuação...

\begin{tabular}{|c|c|c|c|c|c|c|}
\hline Espécies & Famílias & $\mathbf{N}$ & DR & FR & DoR & VI \\
\hline Eriotheca gracilipes (K. Schum.) A. Robyns & Malvaceae & 12 & 1,08 & 1,44 & 1,87 & 4,39 \\
\hline Salvertia convallariodora A. St.-Hil. & Vochysiaceae & 13 & 1,18 & 1,7 & 1,07 & 3,94 \\
\hline Licania humilis Cham. \& Schltdl. & Chrysobalanaceae & 11 & 0,99 & 1,31 & 1,47 & 3,77 \\
\hline Eugenia aurata O. Berg. & Myrtaceae & 13 & 1,18 & 1,7 & 0,69 & 3,56 \\
\hline Machaerium acutifolium Vogel & Fabaceae & 13 & 1,18 & 1,31 & 0,89 & 3,38 \\
\hline Caryocar brasiliense Cambess. & Caryocaraceae & 9 & 0,81 & 1,05 & 1,48 & 3,34 \\
\hline Buchenavia tomentosa Eichler & Combretaceae & 6 & 0,54 & 0,79 & 1,94 & 3,27 \\
\hline Mouriri elliptica Mart. & Melastomataceae & 13 & 1,18 & 1,18 & 0,82 & 3,17 \\
\hline Erythroxylum suberosum A. St.-Hil. & Erythroxylaceae & 14 & 1,27 & 1,05 & 0,59 & 2,9 \\
\hline Hymenaea stigonocarpa Mart. ex Hayne & Fabaceae & 9 & 0,81 & 1,05 & 0,66 & 2,52 \\
\hline Connarus suberosus Planch. & Connaraceae & 10 & 0,9 & 1,05 & 0,47 & 2,42 \\
\hline Annona coriacea Mart. & Annonaceae & 7 & 0,63 & 0,79 & 0,86 & 2,28 \\
\hline Emmotum nitens (Benth.) Miers. & Icacinaceae & 8 & 0,72 & 1,05 & 0,49 & 2,26 \\
\hline Curatella americana $\mathrm{L}$. & Dilleniaceae & 7 & 0,63 & 0,79 & 0,83 & 2,25 \\
\hline Handroanthus ochraceus (Cham.) Mattos & Bignoniaceae & 6 & 0,54 & 0,79 & 0,9 & 2,23 \\
\hline Couepia grandiflora (Mart. \& Zucc.) Benth. \& Hook. f. & Chrysobalanaceae & 8 & 0,72 & 0,92 & 0,52 & 2,16 \\
\hline Ouratea spectabilis (Mart. ex Engl.) Engl. & Ochnaceae & 7 & 0,63 & 0,79 & 0,74 & 2,16 \\
\hline Anacardium occidentale L. & Anacardiaceae & 6 & 0,54 & 0,79 & 0,69 & 2,02 \\
\hline Salacia crassifolia (Mart. ex Schult.) G. Don. & Celastraceae & 6 & 0,54 & 0,79 & 0,62 & 1,95 \\
\hline Pouteria ramiflora (Mart.) Radlk. & Sapotaceae & 4 & 0,36 & 0,52 & 1,03 & 1,91 \\
\hline Mezilaurus crassiramea (Meissn.) Taub. ex Mez & Lauraceae & 6 & 0,54 & 0,79 & 0,56 & 1,89 \\
\hline Vatairea macrocarpa (Benth.) Ducke & Fabaceae & 7 & 0,63 & 0,79 & 0,44 & 1,86 \\
\hline Peltogyne confertiflora (Mart. ex Hayne) Benth. & Fabaceae & 5 & 0,45 & 0,65 & 0,58 & 1,69 \\
\hline Erythroxylum tortuosum Mart. & Erythroxylaceae & 6 & 0,54 & 0,79 & 0,3 & 1,62 \\
\hline Mimosa laticifera Rizzini \& A. Mattos & Fabaceae & 6 & 0,54 & 0,79 & 0,25 & 1,58 \\
\hline Callisthene fasciculata Mart. & Vochysiaceae & 5 & 0,45 & 0,52 & 0,25 & 1,23 \\
\hline Aspidosperma multiflorum A. DC. & Apocynaceae & 5 & 0,45 & 0,39 & 0,28 & 1,12 \\
\hline Guapira noxia (Netto) Lundell & Nyctaginaceae & 4 & 0,36 & 0,52 & 0,18 & 1,07 \\
\hline Xylopia aromatica (Lam.) Mart. & Annonaceae & 3 & 0,27 & 0,39 & 0,4 & 1,06 \\
\hline Tabebuia aurea (Manso) Benth. \& Hook. f. ex S. Moore & Bignoniaceae & 3 & 0,27 & 0,39 & 0,36 & 1,02 \\
\hline Luetzelburgia praecox (Harms ex Kuntze) Harms & Fabaceae & 3 & 0,27 & 0,39 & 0,33 & 0,99 \\
\hline Leptolobium dasycarpum Vogel & Fabaceae & 3 & 0,27 & 0,39 & 0,24 & 0,91 \\
\hline Myrcia camapuanensis N. Silveira & Myrtaceae & 4 & 0,36 & 0,39 & 0,15 & 0,91 \\
\hline Tachigali aurea Tul. & Fabaceae & 3 & 0,27 & 0,39 & 0,23 & 0,89 \\
\hline Heisteria ovata Benth. & Olacaceae & 2 & 0,18 & 0,26 & 0,29 & 0,74 \\
\hline Simarouba versicolor A. St.-Hil. & Simaroubaceae & 2 & 0,18 & 0,26 & 0,21 & 0,65 \\
\hline Dypterix alata Vogel & Fabaceae & 2 & 0,18 & 0,26 & 0,18 & 0,62 \\
\hline Pterodon pubescens (Benth.) Benth. & Fabaceae & 1 & 0,09 & 0,13 & 0,4 & 0,62 \\
\hline Rourea induta Planch. & Connaraceae & 3 & 0,27 & 0,26 & 0,09 & 0,62 \\
\hline Vernonia ferruginea Less. & Asteraceae & 2 & 0,18 & 0,26 & 0,18 & 0,62 \\
\hline Casearia sylvestris $\mathrm{Sw}$. & Salicaceae & 2 & 0,18 & 0,26 & 0,11 & 0,55 \\
\hline Styrax camporum Pohl & Styracaceae & 2 & 0,18 & 0,26 & 0,09 & 0,53 \\
\hline Tocoyena formosa (Cham. \& Schltdl.) K. Schum. & Rubiaceae & 2 & 0,18 & 0,26 & 0,09 & 0,53 \\
\hline Cybianthus detergens Mart. ** & Myrsinaceae & 2 & 0,18 & 0,26 & 0,05 & 0,49 \\
\hline Cordiera sessilis (Vell.) Kuntze ** & Rubiaceae & 2 & 0,18 & 0,13 & 0,05 & 0,36 \\
\hline Matayba guianensis Aubl. & Sapindaceae & 2 & 0,18 & 0,13 & 0,04 & 0,36 \\
\hline Plenckia populnea Reissek & Celastraceae & 1 & 0,09 & 0,13 & 0,11 & 0,34 \\
\hline Andira cujabensis Benth. & Fabaceae & 1 & 0,09 & 0,13 & 0,09 & 0,32 \\
\hline Brosimum gaudichaudii Trécul & Moraceae & 1 & 0,09 & 0,13 & 0,06 & 0,28 \\
\hline Himatanthus obovatus (Müll. Arg.) Woodson & Apocynaceae & 1 & 0,09 & 0,13 & 0,03 & 0,26 \\
\hline Odontadenia lutea (Vell.) Markgr. ** & Apocynaceae & 1 & 0,09 & 0,13 & 0,04 & 0,26 \\
\hline Byrsonima verbascifolia (L.) DC. ** & Malpighiaceae & 1 & 0,09 & 0,13 & 0,02 & 0,25 \\
\hline Erythroxylum engleri $\mathrm{O}$. E. Schulz. ** & Erythroxylaceae & 1 & 0,09 & 0,13 & 0,02 & 0,24 \\
\hline \multirow[t]{2}{*}{ Erythroxylum testaceum Peyr. ** } & Erythroxylaceae & 1 & 0,09 & 0,13 & 0,02 & 0,24 \\
\hline & & 1.106 & 100 & 100 & 100 & 300 \\
\hline
\end{tabular}


A distribuição dos indivíduos nas classes diamétricas não diferiu entre os inventários (KS, $\chi^{2}=2,23 ; p>0,05$ ) e seguiu o modelo de distribuição de J-reverso, apresentando a maioria dos indivíduos na primeira classe (70,5\% em 2002 e 67,2\% em 2006) (Figura 1a). A distribuição da área basal também não foi alterada no período avaliado (KS, $\left.\chi^{2}=0,04 ; p>0,05\right)$ e foi maior na primeira classe, com queda gradual nas classes seguintes (Figura 1b). Apesar disso, ocorreram mudanças dentro e entre as classes de diâmetro, caracterizando o egresso e o ingresso de indivíduos (Figuras 1c, d).

\section{Parâmetros de dinâmica}

Entre 2002 e 2006 morreram 106 indivíduos, resultando em taxa de mortalidade média anual de 4,01\% ano ${ }^{-1}$ (Tabela 2). O número de indivíduos mortos $\left(\mathrm{r}^{2}=-0,9132 ; p=0,0015\right)$ e a área basal morta $\left(\mathrm{r}^{2}=-0,8625 ; p=0,0058\right)$ apresentaram correlação negativa e significativa com as classes de diâmetro, de modo que a primeira classe diamétrica compreendeu cerca de $80 \%$ dos indivíduos mortos e aproximadamente $40 \%$ da área basal morta no período (Figura 1c, d). No cerrado estudado, as espécies que mais contribuíram para mortalidade nas primeiras classes de diâmetro foram as palmeiras Syagrus comosa $\left(34 \%\right.$ ano $\left.^{-1}\right)$ e S. flexuosa $\left(10,2 \%\right.$ ano $\left.^{-1}\right)$ e as árvores Lafoensia pacari (13,6\% ano $^{-1}$ ) e Aspidosperma tomentosum (11,3\% ano ${ }^{-1}$ ) (Tabela 3).

A taxa de recrutamento da comunidade $\left(6,67 \%\right.$ ano $\left.^{-1}\right)$ excedeu à de mortalidade. $\mathrm{O}$ número de indivíduos recrutados $\left(\mathrm{r}^{2}=-0,7910\right.$; $p=0,0193)$ e a área basal dos recrutas $\left(\mathrm{r}^{2}=-0,7910 ; p=0,0193\right)$ apresentaram correlação negativa e significativa com as classes de diâmetro, de modo que a primeira classe diamétrica contemplou a grande maioria dos indivíduos $(95,2 \%)$ e da área basal $(93,2 \%)$ recrutada no período (Figuras 1c, d). As espécies que mais contribuíram para a elevada taxa de recrutamento foram Syagrus comosa $\left(25,3 \% \mathrm{ano}^{-1}\right)$, S. flexuosa $\left(12,5 \%\right.$ ano $\left.^{-1}\right)$, Aspidosperma tomentosum $\left(10,9 \%\right.$ ano $\left.^{-1}\right) \mathrm{e}$ Qualea multiflora $\left(10,9 \%\right.$ ano $\left.^{-1}\right)$.

Tabela 2. Parâmetros de dinâmica expressos em número de indivíduos e em área basal da comunidade lenhosa em uma área de Cerrado Típico amostrada no Parque do Bacaba, Nova Xavantina, MT, entre 2002 (Marimon-Junior \& Haridasan 2005) e 2006.

Table 2. Dynamics parameters expressed in number of individuals and basal area of the woody community of an area of typical cerrado sampled in the Bacaba Park, Nova Xavantina, Mato Grosso State, between 2002 (Marimon-Junior \& Haridasan 2005) and 2006.

\begin{tabular}{lrr}
\hline \multicolumn{1}{c}{ Parâmetros } & Número de indivíduos & Área basal \\
\hline Taxas anuais médias de mortalidade ( $\mathrm{n}^{\circ}$ de ind.) e perda (área basal) $\left(\%\right.$ ano $\left.^{-1}\right)$ & 4,01 & 0,68 \\
Taxas anuais médias de recrutamento ( ${ }^{\circ}$ de ind.) e ganho (área basal) $\left(\%\right.$ ano $\left.^{-1}\right)$ & 6,67 & 2,27 \\
Tempo de meia vida (anos) & 58,15 & 118,32 \\
Tempo de duplicação (anos) & 11,14 & 34,65 \\
Reposição (anos) & 47,60 \\
Estabilidade (anos) & 47,01 & 66,06 \\
Taxa de mudança & 4,00 & 64,52 \\
\hline
\end{tabular}

Tabela 3. Parâmetros de dinâmica (em número de indivíduos) das populações das principais espécies lenhosas ( $\mathrm{n} \geq 13$ ), incremento periódico anual em diâmetro (IPA, $\left.\mathrm{cm} \mathrm{ano}^{-1}\right)$ e coeficiente de variação $(\mathrm{CV}, \%)$ em uma área de Cerrado Típico amostrada no Parque do Bacaba, Nova Xavantina-MT no período de quatro anos (2002 a 2006), sendo: $m=$ taxa anual média de mortalidade $\left(\%\right.$ ano $\left.{ }^{-1}\right) ; r=$ taxa anual média de recrutamento $\left(\%\right.$ ano $\left.{ }^{-1}\right) ; \mathrm{t}_{1 / 2}=$ tempo de meia vida $($ anos $) ;$ $\mathrm{t}_{2}=$ tempo de duplicação (anos); e mu = taxa de mudança das espécies (anos).

Table 3. Dynamics parameters (in number of individuals) of the populations of principal woody species ( $\mathrm{n} \geq 13$ ), periodic annual increment in diameter (IPA, $\mathrm{cm}$ year $\left.^{-1}\right)$ and coefficient of variation $(\mathrm{CV}, \%)$ in an area of typical cerrado sampled in the Bacaba Park, Nova Xavantina-MT over four-year period (2002 to 2006), where: $m=$ mortality rate $\left(\%\right.$ year $\left.{ }^{-1}\right), r=$ recruitment rate $\left(\%\right.$ year $\left.{ }^{-1}\right), \mathrm{t}_{1 / 2}=$ half life time (years), $\mathrm{t}_{2}=$ doubling time $($ years $)$; and mu $=$ species change rate

\begin{tabular}{|c|c|c|c|c|c|c|c|c|}
\hline Espécies & Famílias & m & $\mathbf{r}$ & $t_{1 / 2}$ & $\mathbf{t}_{2}$ & mu & IPA & CV \\
\hline Qualea parviflora & Vochysiaceae & 3,01 & 4,12 & 55,71 & 17,21 & 3,01 & 0,32 & 62,15 \\
\hline Davilla elliptica & Dilleniaceae & 1,01 & 3,83 & 23,88 & 19,75 & 1,01 & 0,18 & 113,17 \\
\hline Roupala montana & Proteaceae & 6,16 & 7,77 & 32,78 & 9,20 & 6,16 & 0,42 & 65,68 \\
\hline Syagrus flexuosa & Arecaceae & 10,24 & 12,55 & 18,91 & 5,79 & 10,24 & 0,35 & 207,24 \\
\hline Aspidosperma tomentosum & Apocynaceae & 11,32 & 10,98 & 76,23 & 6,13 & 11,32 & 0,22 & 77,26 \\
\hline Qualea grandiflora & Vochysiaceae & 2,13 & 2,81 & 92,87 & 24,92 & 2,13 & 0,26 & 72,38 \\
\hline Byrsonima pachyphylla & Malpighiaceae & 8,12 & 9,09 & 40,18 & 7,70 & 8,12 & 0,20 & 87,86 \\
\hline Qualea multiflora & Vochysiaceae & 9,89 & 10,95 & 31,86 & 6,40 & 9,89 & 0,32 & 68,87 \\
\hline Guapira graciliflora & Nyctaginaceae & 9,04 & 9,91 & 38,79 & 7,03 & 9,04 & 0,40 & 75,77 \\
\hline Euplassa inaequalis & Proteaceae & 3,51 & 1,98 & 12,42 & 42,96 & $-3,51$ & 0,72 & 76,41 \\
\hline Vochysia rufa & Vochysiaceae & 1,98 & 0,00 & 34,63 & 0,00 & $-1,98$ & 0,23 & 74,96 \\
\hline Magonia pubescens & Sapindaceae & 2,02 & 3,01 & 65,14 & 23,53 & 2,02 & 0,34 & 115,93 \\
\hline Astronium fraxinifolium & Anacardiaceae & 3,55 & 3,43 & 0,00 & 19,83 & 3,55 & 0,33 & 52,30 \\
\hline Kielmeyera rubriflora & Clusiaceae & 4,45 & 1,27 & 15,20 & 67,91 & $-4,45$ & 0,49 & 94,31 \\
\hline Syagrus comosa & Arecaceae & 33,97 & 25,36 & 0,00 & 2,36 & 33,97 & 0,73 & 70,10 \\
\hline Myrcia lanuginosa & Myrtaceae & 0,00 & 10,37 & 6,32 & 9,12 & 0,00 & 0,14 & 105,93 \\
\hline Ouratea hexasperma & Ochnaceae & 3,01 & 0,00 & 22,61 & 0,00 & $-3,01$ & 0,23 & 95,35 \\
\hline Pseudobombax longiflorum & Malvaceae & 0,00 & 2,90 & 23,53 & 26,31 & 0,00 & 0,26 & 120,96 \\
\hline Kielmeyera speciosa & Clusiaceae & 1,52 & 1,50 & 42,96 & 45,73 & 1,52 & 0,33 & 56,11 \\
\hline Lafoensia pacari & Lythraceae & 13,62 & 10,20 & 0,00 & 6,03 & 13,62 & 0,21 & 71,40 \\
\hline
\end{tabular}


O tempo de meia vida da comunidade estudada foi maior que o tempo de duplicação, considerando tanto os indivíduos como a área basal (Tabela 2). Este resultado também foi observado para a maioria das espécies avaliadas, o que resultou em mudanças predominantemente positivas para as mesmas (Tabela 3 ). A reposição (turnover) indicou que a comunidade é mais dinâmica em relação ao número de indivíduos (34,6 anos) do que em relação à área basal (86 anos) (Tabela 2). Quanto à estabilidade, a comunidade estudada é mais estável em termos de número de indivíduos (47 anos) do que em relação à área basal (64,5 anos) (Tabela 2). Por outro lado, a mudança foi mais elevada para área basal (5,5 anos) do que para o número de indivíduos (4 anos) (Tabela 2).

O IPA da comunidade, considerando todos os indivíduos de todas as espécies em um período de quatro anos (2002 a 2006), foi de $0,31 \mathrm{~cm}^{2}$ ano $^{-1}$ (desvio padrão $=0,32$, mediana $=0,25$ e coeficiente de variação $=103,17 \%)$. O IPA da comunidade apresentou correlação positiva e significativa com as classes de diâmetro $\left(r^{2}=0,8929\right.$; $p=0,0068$ ), sendo mais elevado em indivíduos de maior porte. Entre as espécies que apresentaram os maiores IPAs estão Euplassa inaequalis $\left(0,72 \mathrm{~cm} \mathrm{ano}^{-1}\right)$, Kielmeyera rubriflora $\left(0,49 \mathrm{~cm}^{2} \mathrm{ano}^{-1}\right)$ e Roupala montana $\left(0,42 \mathrm{~cm} \mathrm{ano}^{-1}\right)$. O coeficiente de variação do IPA das espécies avaliadas apresentou elevada variação (Tabela 3 ).

\section{Discussão}

\section{Composição florística e diversidade de espécies}

As alterações temporais observadas na composição florística da comunidade estudada estão relacionadas ao aparecimento e ao desaparecimento de alguns taxa. No período (2002 a 2006), o número de espécies novas foi superior ao de espécies desaparecidas, o que resultou em balanço positivo para a comunidade, como também verificado por Henriques \& Hay (2002) em área de Cerrado sentido restrito protegida do fogo há 37 anos no Distrito Federal. Ao contrário, Felfili et al. (2000) constataram um balanço negativo em um cerrado submetido ao fogo no Distrito Federal, onde o desaparecimento de espécies foi superior ao surgimento. Hoffmann \& Moreira (2002) e Aquino et al. (2007b) observaram que em Cerrado sentido restrito muitas espécies podem desaparecer após um ou mais eventos de queimada. No caso do cerrado estudado, o balanceamento positivo pode ter sido reflexo da recomposição das espécies na área após o evento de queimada ocorrido no ano de 2001.

Convém salientar que no presente estudo o aparecimento e o desaparecimento de espécies se limitaram às de baixa abundância ( $\mathrm{N} \leq 2$ indivíduos), fato também constatado por Felfili et al. (2000), Hoffmann \& Moreira (2002), Libano \& Felfili (2006) e por Aquino et al. (2007b) em áreas de Cerrado sentido restrito. A vegetação de cerrado frequentemente apresenta um grupo de espécies que prevalece em termos de densidade e área basal sobre as demais (Felfili \& Felfili 2001, Assunção \& Felfili 2004). No entanto, geralmente são registradas muitas espécies com baixa abundância, caracterizadas como raras (Assunção \& Felfili 2004). As espécies raras são muito suscetíveis ao surgimento e desaparecimento em uma mesma área, pois qualquer flutuação nas taxas de mortalidade ou recrutamento pode ser determinante para a definição de presença ou ausência de tais espécies na comunidade (Felfili et al. 2000, Aquino et al. 2007b). Nesse sentido, o aumento temporal da riqueza no presente estudo pode ser considerado transitório, como também sugerido por Aquino et al. (2007b) para um cerrado no Nordeste do Brasil.
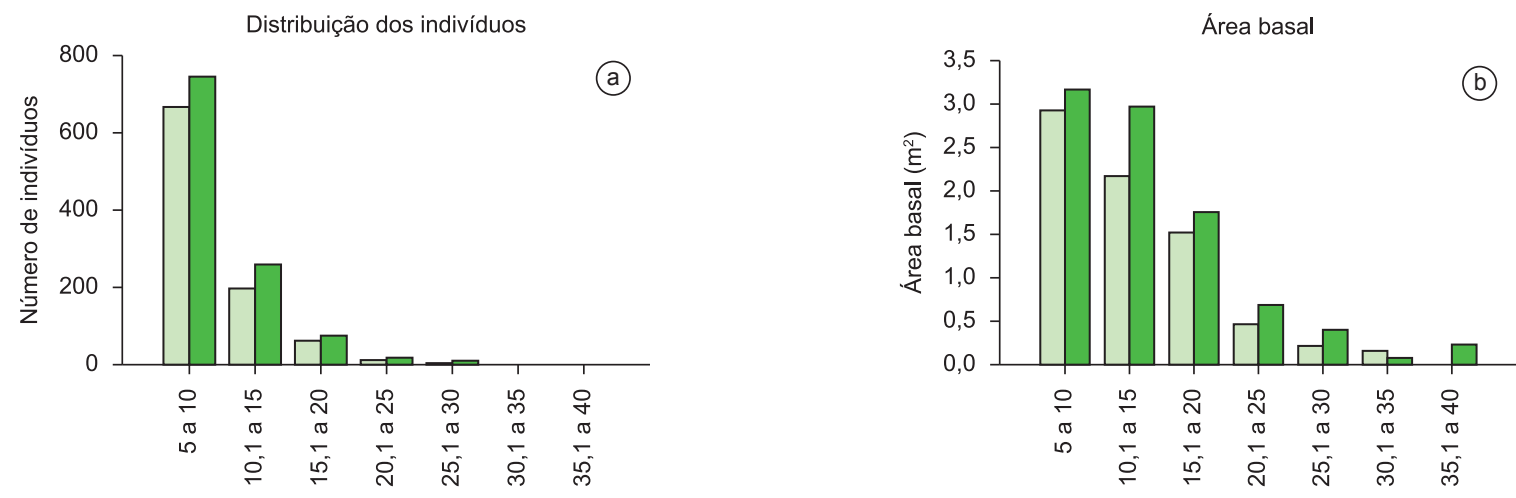

$\square 2002 \square 2006$

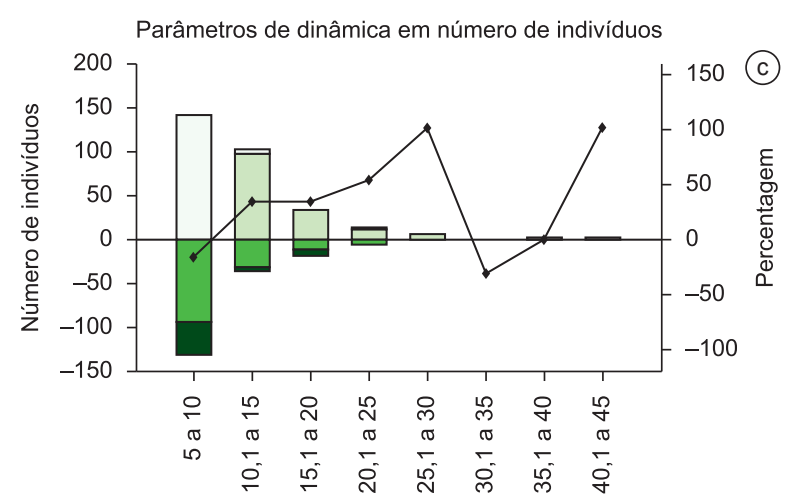

Classes de diâmetro $(\mathrm{cm})$

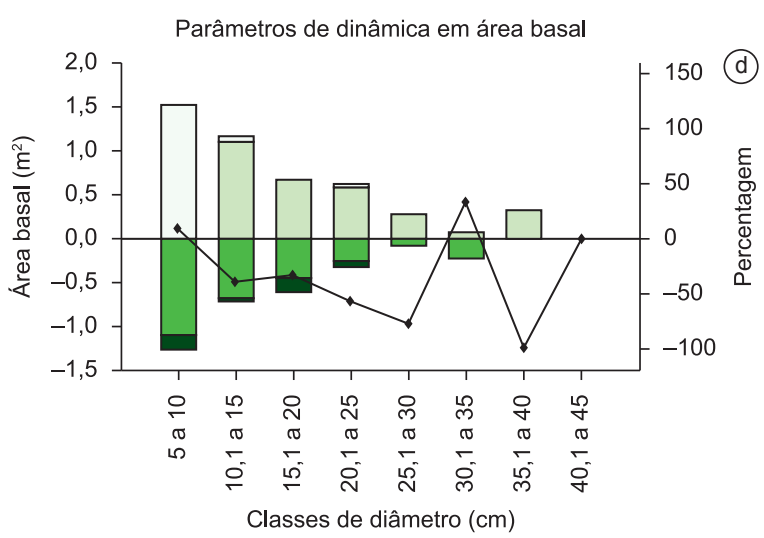

- Morto $\square$ Egresso $\square$ Recruta $\square$ Ingresso Mudança

Figura 1. Mudanças estruturais e dinâmica da comunidade lenhosa por classe de diâmetro do Cerrado Típico de Nova Xavantina, MT.

Figure 1. Structural changes and dynamics of the woody community per diameter classes in a typical cerrado in Nova Xavantina, MT. 
A posição de destaque de Fabaceae entre as famílias com maior riqueza de espécies e a manutenção deste padrão ao longo do período de estudo podem estar relacionados com a capacidade de fixação de nitrogênio de algumas de suas espécies, o que pode conferir à referida família vantagem competitiva nos solos predominantemente distróficos do Cerrado (Goodland 1979). Além disso, a grande representatividade de Fabaceae pode ser atribuída ao fato da região central do Brasil ser considerada o principal centro de diversificação desta família (Polhill et al. 1981). Por outro lado, o sucesso de Vochysiaceae pode estar fortemente associado à capacidade de acumulação de alumínio por parte de algumas das suas espécies (Haridasan \& Araújo 1987, Haridasan 2000), o que também configura uma vantagem competitiva em solos distróficos com baixa saturação de bases trocáveis, baixa capacidade de troca catiônica, alta acidez e altas concentrações de $\mathrm{Al}$ no perfil, como é o caso da maioria dos solos no bioma Cerrado. Além disso, muitas espécies das referidas famílias têm estratégias reprodutivas bem adaptadas às condições climatológicas do Cerrado, apresentando dispersão das sementes no início da estação chuvosa, boa germinação e ausência de dormência nas sementes (Oliveira 2008).

A diversidade de espécies registrada neste estudo foi elevada e semelhante à encontrada por outros autores em áreas de Cerrado sentido restrito (Marimon et al. 1998, Felfili et al. 2000, Nogueira et al. 2001, Fidelis \& Godoy 2003, Assunção \& Felfili 2004). A manutenção temporal da riqueza e diversidade de espécies, observadas no presente estudo, parece ser um padrão esperado nas formações savânicas do Cerrado, visto que também foi registrada em outros estudos em Cerrado sentido restrito, tal como o de Aquino et al. (2007b). Os valores da equabilidade sugerem que os indivíduos estiveram distribuídos de forma razoavelmente equânime entre as espécies no período avaliado, caracterizando baixa dominância ecológica. No entanto, a elevada densidade de algumas espécies, como Qualea parviflora e Davilla elliptica (Tabela 1), contribuiu para uma limitação do valor deste parâmetro em todo período estudado.

\section{Estrutura da comunidade}

O desbalanceamento positivo no número de indivíduos registrados em 2002 e 2006 pode ser atribuído, principalmente, ao aumento da densidade de Aspidosperma tomentosum (35\%), Syagrus flexuosa (32\%) e Byrsonima pachyphylla (27\%), que parecem ser espécies boas competidoras no cerrado estudado. Por outro lado, a elevada taxa de mortalidade de algumas espécies, como Lafoensia pacari (Tabela 3), contribuiu para a perda de biomassa da comunidade lenhosa (Tabela 2). Contudo, o aumento na densidade e a reduzida taxa de mortalidade em relação à de recrutamento em algumas das espécies de maior densidade (Tabela 3 ) elevaram a taxa anual média de ganho de área basal da comunidade, superando a taxa de perda e resultando em balanço positivo no período, caracterizado pela maior biomassa no segundo inventário. Provavelmente esta situação foi ocasionada pela ausência de fogo no período avaliado, o que deve ter proporcionado maior estabelecimento de indivíduos lenhosos e redução da mortalidade de indivíduos de menor porte, resultando em aumento da densidade e biomassa total da comunidade, processo também observado por Moreira (2000), Henriques \& Hay (2002) e Hoffmann \& Moreira (2002) em cerrados do Distrito Federal.

Moreira (2000), comparando duas áreas, uma sujeita a queimadas bianuais e outra protegida do fogo há quase 30 anos, observou que Roupala montana e Aspidosperma tomentosum ( $3^{\mathrm{a}}$ e $5^{\mathrm{a}}$ de maior VI no presente estudo, respectivamente), estiveram entre as espécies que apresentaram maior densidade na área protegida, podendo ser consideradas sensíveis ao fogo. Emmotum nitens, uma espécie considerada como típica de cerradão (Furley \& Ratter 1988), cuja estrutura radicular impossibilita a rebrota após o fogo (Moreira 2000), também foi registrada na área do presente estudo, o que reforça o padrão esperado em uma área protegida do fogo por um período de quatro anos.
O padrão de distribuição dos indivíduos em classes de diâmetro observado no presente estudo também foi constatado por Felfili et al. (2000), Henriques \& Hay (2002), Fidelis \& Godoy (2003) e Assunção \& Felfili (2004) em outras áreas de Cerrado sentido restrito, indicando comunidade autorregenerativa (Assunção \& Felfili 2004) e com recrutamento contínuo (Felfili et al. 2000). No entanto, o modelo de J-reverso pode apresentar limitação em função da elevada proporção de espécies no Cerrado Típico com potencialidade genética para apresentar porte pequeno, resultando em "estado absorvente" nas menores classes de diâmetro (Scolforo et al. 1998). No caso do cerrado estudado, além da grande concentração de indivíduos nas menores classes, a ausência de classes vazias e a manutenção temporal do padrão de distribuição reforçam a sugestão de que a comunidade está em bom estado de conservação e apresentando reposição contínua de indivíduos em função de uma reprodução satisfatória (Silva-Júnior \& Silva 1988, Felfili et al. 2000)

As espécies referidas como principais em Valor de Importância no presente estudo também foram apontadas por Felfili \& Silva-Júnior (1988), Marimon et al. (1998), Nogueira et al. (2001), Ratter et al. (2003) e Balduino et al. (2005) como de grande representatividade e ampla distribuição no bioma. Além disso, o elevado VI e a manutenção temporal deste parâmetro podem estar relacionados à elevada plasticidade apresentada por muitas espécies de cerrado (Hoffmann \& Franco 2003), indicando que suas posições de destaque na estrutura da comunidade poderão ser mantidas ao longo do tempo, caso não ocorram interferências severas, tais como a entrada frequente de fogo.

\section{Parâmetros de dinâmica}

Entre 2002 e 2006, a taxa de mortalidade foi superada pela de recrutamento, resultando em balanço positivo para a comunidade. De acordo com Corrêa \& Van Den Berg (2002), quando a taxa de recrutamento excede à de mortalidade, a taxa de mudança é positiva para as populações, indicando equilíbrio dinâmico em relação à recomposição da comunidade. No caso da comunidade estudada, a ausência de incêndios no período avaliado pode ter sido fator determinante para o expressivo estabelecimento de novos indivíduos, resultando em taxa elevada de recrutamento $\left(6,67 \% \mathrm{ano}^{-1}\right)$. A grande capacidade de rebrota a partir de caules e raízes e os outros tipos de reprodução vegetativa inerentes a muitas espécies do Cerrado sentido restrito certamente contribuíram para garantir a elevada taxa de recrutamento do cerrado avaliado, como também observado por Hoffmann \& Moreira (2002), Henriques \& Hay (2002) e Aquino et al. (2007b).

De acordo com Hoffmann \& Moreira (2002), quando uma população não é exposta a queimadas por períodos superiores a três anos, grandes indivíduos adultos podem ser comuns e a população tende a recrutar a taxas superiores a $5 \%$ ano $^{-1}$, tal como verificado para a comunidade e muitas populações avaliadas no presente estudo. Neste caso, os referidos autores observaram ainda que, se a população fica exposta a queimadas trienais, os indivíduos adultos podem produzir numerosos brotos que representam aumento significativo na densidade dos indivíduos. Contudo, depois de repetidas queimadas, a população passa a ser dominada por indivíduos de pequeno porte e pode entrar em declínio (Felfili et al. 2000, Libano e Felfili 2006).

Tal como verificado no presente estudo, Henriques \& Hay (2002) e Aquino et al. (2007b) também registraram maior mortalidade em indivíduos de pequeno porte. Segundo Henriques \& Hay (2002), Miranda et al. (2002) e Miranda \& Sato (2005) este padrão está relacionado à maior suscetibilidade dos indivíduos menores aos efeitos do fogo. Para os referidos autores, algumas adaptações como a espessura da casca, presença de órgãos subterrâneos de reserva e tamanho crítico de escape ao fogo podem não estar bem desenvolvidos em indivíduos jovens, favorecendo a mortalidade. 
Por outro lado, o recrutamento de indivíduos também ocorreu preferencialmente nas menores classes de diâmetro, resultando em reposição dos indivíduos de pequeno porte na comunidade. As espécies que mais contribuíram para o expressivo número de indivíduos mortos nas classes iniciais de diâmetro também apresentaram elevadas taxas de recrutamento no período, corroborando o padrão observado por Felfili (1995) para as espécies arbóreas de uma mata de galeria. A referida autora destacou que as espécies mais abundantes estão sujeitas às maiores taxas de mortalidade e recrutamento, justamente por apresentarem elevada densidade de indivíduos. No caso da comunidade estudada, este padrão indica que as posições de destaque de tais espécies na estrutura da comunidade serão mantidas ao longo do tempo, caso as mesmas continuem apresentando reprodução satisfatória, como também observado por Felfili (1995) e Aquino et al. (2007a) em mata de galeria e Cerrado sentido restrito, respectivamente.

Algumas espécies consideradas comuns na área, como Vochysia rufa e Ouratea hexasperma, não apresentaram taxas de recrutamento suficientemente altas para compensar às de mortalidade, o que pode ser indicativo de que a estrutura de tais populações poderá sofrer mudanças negativas, caso o atual padrão seja mantido. Apesar disso, Aquino et al. (2007b) observaram que a manutenção das características estruturais pode ocorrer em espécies de reduzido recrutamento quando tais espécies apresentam elevada sobrevivência. No caso da comunidade estudada, de forma geral, as populações mais importantes mantiveram sua estrutura, proporcionando acúmulo de indivíduos e de biomassa para a comunidade como um todo. Além disso, não se pode descartar a possibilidade das referidas espécies apresentarem recrutamento episódico, com períodos de elevado recrutamento, devido ao surgimento de condições ótimas temporárias, intercalados por períodos de reduzido recrutamento (Fordyce et al. 2000, Marimon \& Felfili 2006).

O valor do IPA registrado para a comunidade do presente estudo foi superior ao encontrado por Aquino et al. (2007b) em dois fragmentos de cerrado no Maranhão $\left(0,13\right.$ e $\left.0,17 \mathrm{~cm}^{2} \mathrm{ano}^{-1}\right)$ e por Henriques \& Hay (2002) em um cerrado no Distrito Federal $\left(0,16 \mathrm{~cm}^{2} \mathrm{ano}^{-1}\right)$. Henriques $\&$ Hay (2002) sugeriram que a baixa taxa de incremento da parte aérea das plantas do Cerrado sentido restrito pode estar associada com solos pobres em nutrientes e com os períodos prolongados de seca. Nesse sentido, a diferença entre o IPA da comunidade estudada e o incremento registrado nas áreas comparadas pode ter ocorrido em resposta às possíveis diferenças na fertilidade ou textura do solo e/ou às peculiares condições de preservação de cada área. O elevado valor do coeficiente de variação do IPA apresentado pela comunidade aqui estudada sugere a presença de espécies com grande plasticidade em responder às variações ambientais, o que, neste caso, reforça a opinião de que o cerrado apresenta maior resiliência do que outras formações vegetais (Hoffmann \& Franco 2003).

O aumento gradual do IPA ao longo das classes de diâmetro corroborou com o padrão de incremento descrito por Arce et al. (2000) para Cerrado sentido restrito. Os referidos autores observaram que indivíduos lenhosos de maior porte apresentaram crescimento relativo mais elevado que os de menor porte. Neste caso, indivíduos de maior porte geralmente apresentam raízes e outros órgãos de reserva mais desenvolvidos do que indivíduos menores (Sperry et al. 1998), o que pode lhes conferir maior vantagem em produção relativa de biomassa e, em consequência, maior incremento em diâmetro.

A análise da dinâmica das principais espécies apontou Qualea parviflora, Roupala montana, Syagrus flexuosa, Magonia pubescens e Qualea multiflora como espécies de reduzida mortalidade e de recrutamento e crescimento relativamente elevados no período. Estes resultados indicam que estas espécies podem apresentar potencial para restauração e manejo de áreas de cerrado na região Nordeste de Mato Grosso, destacando a necessidade da realização de estudos mais detalhados (testes de germinação e crescimento em viveiro e em campo) para confirmar esta recomendação. Outras espécies, como Kielmeyera rubriflora e Euplassa inaequalis, também apresentaram crescimento relativamente elevado no período. No entanto, a relação mortalidade/recrutamento favoreceu a mortalidade, sugerindo que estudos populacionais mais específicos são necessários para avaliar as possíveis causas desta mortalidade antes de recomendações de uso destas espécies em estratégias de recuperação.

Em resposta ao desbalanceamento positivo entre as taxas anuais médias de mortalidade e recrutamento, o tempo de meia vida superou o tempo de duplicação, tanto para a comunidade quanto para a maioria das espécies avaliadas, constituindo um padrão de mudanças positivas no período. A comunidade estudada apresentou elevada reposição e estabilidade, mantendo a riqueza e a estrutura da comunidade no período de estudo e indicando equilíbrio dinâmico em relação à recomposição, como também sugerido por Aquino et al. (2007b) para um Cerrado sentido restrito. Neste caso, tais padrões podem estar relacionados à ausência de fogo no período estudado, o que estaria ocasionando reduzida mortalidade e elevado estabelecimento de novos indivíduos. Estes resultados sugerem que a comunidade estudada está apresentando boa resiliência aos distúrbios causados pela entrada de fogo em períodos anteriores (2001), pois as mudanças foram positivas no período subsequente (2002 a 2006).

Os padrões de dinâmica da comunidade e das espécies avaliadas também sugerem que mudanças ocorreram na composição florística e na estrutura da comunidade, caracterizadas pelo balanço positivo entre a entrada e saída de algumas espécies e pelo aumento da densidade de indivíduos e biomassa. As mudanças positivas aqui registradas podem estar relacionadas à ausência de fogo durante o período estudado, condição que favoreceu o estabelecimento de muitos indivíduos, compensando a taxa anual média de mortalidade entre 2002 e 2006.

\section{Agradecimentos}

Ao Laboratório de Ecologia Vegetal da Universidade do Estado de Mato Grosso - UNEMAT, Campus de Nova Xavantina, pelo material cedido e pelo apoio logístico durante a etapa de campo.

\section{Referências Bibliográficas}

ABAD, J.C.S. \& MARIMON, B.S. 2008. Caracterização e diagnóstico ambiental do Parque Municipal do Bacaba (Nova Xavantina, MT). In Gestão e educação ambiental: água, biodiversidade e cultura (J.E. Santos \& C. Galbiati, orgs.). Rima, São Carlos, p.23-56.

ANGIOSPERM PHYLOGENY GROUP - APG. 2009. An update of the Angiosperm Phylogeny Group classification for the orders and families of flowering plants: APG III. Bot. J. Linn. Soc. 161:105-121.

AQUINO, F.G., WALTER, B.M.T. \& RIBEIRO, J.F. 2007a. Dinâmica de populações de espécies lenhosas de Cerrado, Balsas, Maranhão. Rev. Árvore 31(5): 793-803.

AQUINO, F.G., WALTER, B.M.T. \& RIBEIRO, J.F. 2007b. Woody community dynamics in two fragments of "cerrado" stricto sensu over a seven-year period (1995-2002), MA, Brazil. Revista Brasil. Bot. 30(1):113-121.

ARCE, J.E., FELFILI, J.A., GAIAD, D., REZENDE, A.V. \& SANQUETTA, C.R. 2000. Avaliação do crescimento da vegetação arbórea em uma área de cerrado sensu stricto, em Brasília, DF. In Colégio de ingenieros del Peru. Capítulos de Ingenieria florestal (I. Lombardi, org.). Congresso Florestal Latinoamericano. Tomo III.

ASSUNÇÃO, S.L. \& FELFILI, J.M. 2004. Fitossociologia de um fragmento de cerrado sensu stricto na APA do Paranoá, DF, Brasil. Acta Bot. Bras. 18(4):903-909. 
BALDUINO, A.P.C., SOUZA, A.L., MEIRA NETO, J.A.A., SILVA, A.F \& SILVA-JÚNIOR, M.C. 2005. Fitossociologia e análise comparativa da composição florística do cerrado de Paraopeba-MG. Rev. Árvore 29(1):25-34.

BONINI, E.E. \& BONINI, S.E. 1972. Estatística teórica e exercícios. Editora Loyola, São Paulo.

BROWER, J.E. \& ZAR, J.H. 1977. Field and laboratory methods for general ecology. W.C. Brown Co. Pub., Iowa.

CORRÊA, B.S. \& VAN DEN BERG, E. 2002. Estudo da dinâmica da população de Xylopia brasiliensis Sprengel em relação a parâmetros populacionais e da comunidade em uma floresta de galeria em Itutinga, MG, Brasil. Cerne 8(1):01-12.

ENCINAS, J.M.I., SILVA, G.F. \& PINTO, J.R.R. 2005. Idade e crescimento das árvores. Comun. Técn. Fl. da UnB 7(1):1-40.

FELFILI, J.M. 1995. Growth, recruitment and mortality in the Gama gallery forest in central Brazil over a six-year period (1985-1991). J. Trop. Ecol. 11:67-83.

FELFILI, J.M. \& SILVA Jr., M.C. 1988. Distribuição dos diâmetros numa faixa de Cerrado na Fazenda Água Limpa (FAL) em Brasília-DF. Acta Bot. Bras. 2(1-2):85-104.

FELFILI, J.M., REZENDE, A.V., SILVA Jr., M.C. \& SILVA, M.A. 2000 Changes in the floristic composition of cerrado sensu stricto in Brazil over a nine-year period. J. Trop. Ecol. 16:579-590.

FELFILI, M.C. \& FELFILI, J.M. 2001. Diversidade alfa e beta no cerrado sensu stricto da Chapada Pratinha, Brasil. Acta Bot. Bras. 15(2):243-254.

FIDELIS, A.T. \& GODOY, S.A.P. 2003. Estrutura de um cerrado stricto sensu na Gleba Cerrado Pé-de-Gigante, Santa Rita do Passa Quatro, SP, Brasil. Acta Bot. Bras. 17(4):531-539.

FORDYCE, I.R., EAMUS, D. \& DUFF, G.A. 2000. Episodic seedling growth in Allosyncarpia ternate, a lignotuberous, monsoon rainforest tree in northern Australia. Austral Ecol. 25:25-35.

FURLEY, P.A. \& RATTER, J.A. 1988. Soil resources and plant communities of Central Brazilian cerrado and their development. J. Biogeogr. 15:97-108.

GOODLAND, R.J. 1979. Análise ecológica da vegetação do cerrado. In Ecologia do Cerrado (R.J. Goodland, ed.). USP, São Paulo, p.61-171.

HARIDASAN, M. 2000. Nutrição mineral de plantas nativas do cerrado. Rev. Bras. Fisiol. Veg. 12(1):54-64.

HARIDASAN, M. \& ARAÚJO, G.M. 1987. Aluminium accumulating species in two forest communities in the cerrado region of the central Brazil. Forest Ecol. Manag. 24:15-26.

HENRIQUES, R.P.B. \& HAY, J.D. 2002. Patterns and dynamics of plant populations. In Cerrados of Brazil: ecology and natural history a Neotropical Savanna (P.S. Oliveira \& R.J. Marquis, eds.). Columbia University Press, New York, p.140-158.

HOFFMANN, W.A. \& MOREIRA, A.G. 2002. The role of fire in population dynamics of woody plants. In Cerrados of Brazil: ecology and natural history a Neotropical Savanna (P.S. Oliveira \& R.J. Marquis, eds.). Columbia University Press, New York, p.159-177.

HOFFMANN, W.A. \& FRANCO, A.C. 2003. Comparative growth analysis of tropical forest and savanna woody plants using phylogenetically independent contrasts. J. Ecol. 91:475-484.

KLINK, C.A. \& MACHADO, R.B. 2005. Conservation of the Brazilian Cerrado. Conserv. Biol. 19(3):707-713.

KORNING, J. \& BALSLEV, H. 1994. Growth rates and mortality patterns of tropical lowland tree species and the relation to forest structure in Amazonian Ecuador. J. Trop. Ecol. 10:151-166.

LIBANO, A.M. \& FELFILI, J.M. 2006. Mudanças temporais na composição florística e na diversidade de um cerrado sensu stricto do Brasil Central em um período de 18 anos (1985 - 2003). Acta Bot. Bras. 20(4):927-936.

LIEBERMAN, D., LIEBERMAN, M., PERALTA, R. \& HARTSHORN, S. 1985. Mortality patterns and stand turnover rates in a wet tropical forest in Costa Rica. J. Ecol. 73:915-924.

LUDWING, J.A. \& REYNOLDS, J.E. 1988. Statistical ecology - a primer on methods and computing. J. Wiley \& Sons, New York.
MARIMON, B.S., VARELLA, R.F. \& MARIMON-JUNIOR, B.H. 1998. Fitossociologia de uma área de cerrado de encosta em Nova Xavantina, Mato Grosso. Bol. Herb. Ezechias Paulo Heringer 3(1):82-101.

MARIMON, B.S. \& FELFILI, J.M. 2006. Chuva de sementes em uma floresta monodominante de Brosimum rubescens Taub. e em uma floresta mista adjacente no Vale do Araguaia, MT, Brasil. Acta Bot. Bras. 20(2):423-432.

MARIMON, B.S., LIMA, E.S., DUARTE, T.G., CHIEREGATTO, L.C. \& RATTER, J.A. 2006. Observations on the vegetation of northeastern Mato Grosso, Brazil. IV. An analysis of the Cerrado-Amazonian Forest ecotone. Edinb. J. Bot. 63(2-3):323-341.

MARIMON-JUNIOR, B.H. \& HARIDASAN, M. 2005. Comparação da vegetação arbórea e características edáficas de um cerradão e um cerrado stricto sensu em áreas adjacentes sobre solo distrófico no leste de Mato Grosso, Brasil. Acta Bot. Bras. 19(4):913-926.

MIRANDA, H.S., BUSTAMANTE, M.M.C. \& MIRANDA, A.C. 2002. The fire factor. In Cerrados of Brazil: ecology and natural history a Neotropical Savanna (P.S. Oliveira \& R.J. Marquis, eds.). Columbia University Press, New York, p.51-68.

MIRANDA, H.S. \& SATO, M.N. 2005. Efeitos do fogo na vegetação lenhosa do Cerrado. In Cerrado: ecologia, biodiversidade e conservação (A. Scariot, J.C. Sousa-Silva \& J.M. Felfili, orgs.). Ministério do Meio Ambiente, Brasília, p.93-105.

MINISTÉRIO DO MEIO AMBIENTE, DOS RECURSOS HÍDRICOS E DA AMAZÔNIA LEGAL - MMA. 2009. Monitoramento do desmatamento no bioma Cerrado, 2002 a 2008: dados revisados. Brasília. Relatório técnico.

MISSOURI BOTANICAL GARDEN - MOBOT. http://www.tropicos.org/ (último acesso em 15/01/2010).

MOREIRA, A.G. 2000. Effects of fire protection on savanna structure in Central Brazil. J. Biogeogr. 27:1021-1029.

MÜLLER-DOMBOIS, D. \& ELLEMBERG, H. 1974. Aims and methods of vegetation ecology. J. Wiley \& Sons, New York.

NOGUEIRA, P.E., FELFILI, J.M. \& SILVA Jr., M.C. 2001. Composição florística e fitossociologia do cerrado sentido restrito no município de Canarana-MT. Bol. Herb. Ezechias Paulo Heringer 8:28-43.

OLIVEIRA, P.E.A.M. 2008. Fenologia e Biologia Reprodutiva das Espécies de Cerrado. In Cerrado: ecologia e flora (S.M. Sano, S.P. Almeida \& J.F Ribeiro, eds.). EMBRAPA-CPAC, Planaltina, p.273-290.

OLIVEIRA, R. 2005. The forgotten ecosystem. Nature 437:944-945.

PIVELLO, V.R. 2005. Manejo de fragmentos de Cerrado: princípios para a conservação da biodiversidade. In Cerrado: ecologia, biodiversidade e conservação (A. Scariot, J.C. Sousa-Silva \& J.M. Felfili, orgs.). Ministério do Meio Ambiente, Brasília, p.401-414.

POLHILL, R.M., RAVEN, P.H. \& STIRTON, C.H. 1981. Evolution and systematics of the Leguminosae. In Advances in legume systematics (R.M. Polhill \& P.H. Raven, eds.). Kew Garden.

RATTER, J.A., RIBEIRO, J.F., BRIDGEWATER, S. 1997. The Brazilian Cerrado vegetation and threats to its biodiversity. Ann. Bot. 80:223-230.

RATTER, J.A., BRIDGEWATER, S. \& RIBEIRO, J.F. 2003. Analysis of the floristic composition of the brazilian cerrado vegetation III: comparison of the woody vegetation of 376 areas. Edinb. J. Bot. 60(1):57-109.

RIBEIRO, J.F. \& WALTER, B.M.T. 2008. As principais fitofisionomias do Bioma Cerrado. In Cerrado: ecologia e flora (S.M. Sano, S.P. Almeida \& J.F. Ribeiro, eds.). EMBRAPA-CPAC, Planaltina, p.151-212.

SCHIAVINI, I., RESENDE, J.C.F. \& AQUINO, F.G. 1998. Dinâmica de espécies arbóreas em Mata de Galeria e Mata Mesófila na margem do Ribeirão Panga, MG. In Cerrado: ambiente e flora (S.M. Sano \& S.P. Almeida, eds.). EMBRAPA-CPAC, Planaltina, p.267-299.

SCOLFORO, J.R.S., PULZ, F.A. \& MELO, J.M. 1998. Modelagem da produção, idade das florestas nativas, distribuição espacial das espécies e a análise estrutural. In Manejo Florestal (J.R.S. Scolforo, org.). UFLA; FAEPE, Lavras, p.189-246.

SHEIL, D., BURSLEM, D.F.R.P. \& ALDER, D. 1995. The interpretation and misinterpretation of mortality rate measures. J. Ecol. 83:331-333. 
SHEIL, D., JENNINGS, S. \& SAVILL, P. 2000. Long-term permanent plot observations of vegetation dynamics in Budongo, a Ugandan rain forest. J. Trop. Ecol. 16:765-800.

SIEGEL, S. \& CASTELLAN-JÚNIOR, N.T. 1988. Nonparametric statistics for the behavioral sciences. McGraw-Hill, New York.

SILVA, J.M.C \& BATES, J.M. 2002. Biogeographic patterns and conservation in the South American Cerrado: a Tropical Savanna Hotspot. BioScience 52(3):225-233.

SILVA, F.A.M., ASSAD, E.D. \& EVANGELISTA, B.A. 2008. Caracterização climática do Bioma Cerrado. In Cerrado: ecologia e flora (S.M. Sano, S.P Almeida \& J.F. Ribeiro, eds.). EMBRAPA-CPAC, Planaltina, p.69-88.
SILVA-Jr., M.C. \& SILVA, A.F. 1988. Distribuição dos diâmetros dos troncos das espécies mais importantes do Cerrado na Estação Florestal de Experimentação de Paraopeba (EFLEX)-MG. Acta Bot. Bras. 2(1-2):107-126.

SPERRY, J.S., ADLER, F.R., CAMPBELL, G.S. \& COMSTOCK, J.P. 1998. Limitation of plant water use by rhizosphere and xylem conductance: results from a model. Plant Cell Environ. 21:347-359.

SWAINE, M.D. \& LIEBERMAN, D. 1987. Note on the calculation of mortality rates. J. Trop. Ecol. 3:ii-iii.

WALTER, B.M.T., CARVALHO, A.M. \& RIBEIRO, J.F. 2008. O conceito de Savana e de seu componente Cerrado. In Cerrado: ecologia e flora (S.M. Sano, S.P. Almeida \& J.F. Ribeiro, eds.). EMBRAPA-CPAC, Planaltina, p.19-45.

ZAR, J.H. 1999. Biostatistical analysis. Prentice Hall, New Jersey.

Recebido em 30/06/2010

Versão reformulada recebida em 24/12/2010

Publicado em 24/01/2011 